\title{
Enquêter sur la France au Rwanda en contexte militant
}

\section{Entretien avec François Graner}

\author{
Martin Mourre, Florent Piton, Nathaniel Powell
}

\begin{abstract}
Citer cet article: Mourre Martin, Piton Florent et Powell Nathaniel (2021), « Enquêter sur la France au Rwanda en contexte militant. Entretien avec François Graner », Revue d'Histoire Contemporaine de l'Afrique, Dossier : au-delà du rapport Duclert, 102-117, en ligne. URL :

https://oap.unige.ch/journals/rhca/article/view/rwandamourrepitonpowell
\end{abstract}

Mise en ligne : 08 novembre 2021

DOI : https://doi.org/10.51185/journals/rhca.2021.e583

Cet entretien a été réalisé le 18 mai 2021, quelques semaines après la remise du rapport de la Commission de recherche sur les archives françaises relatives au Rwanda et au génocide des Tutsi (dite commission Duclert, du nom de son président) et avant la visite le 27 mai d'Emmanuel Macron au Rwanda. Membre de longue date de l'association Survie, par ailleurs directeur de recherches en physique, François Graner est l'auteur de deux ouvrages relatifs à l'action française au Rwanda avant, pendant et après le génocide des Tutsi, dont le dernier, co-écrit avec Raphaël Doridant, est paru en 20201. À l'issue d'une procédure de plusieurs années, François Graner a également obtenu, en juin 2020 par une décision contentieuse du Conseil d'État, la levée d'une série de blocages l'empêchant d'accéder aux archives du président Mitterrand sur le Rwanda. Plutôt que de l'interroger, comme cela a été fait dans d'autres revues et d'autres contextes, sur la procédure qui lui a permis d'obtenir cette décision ou sur ses conclusions et celles de Survie quant au rôle de la France au Rwanda, nous avons souhaité revenir avec lui sur les sources mobilisées pour écrire cette histoire de la France au Rwanda. Cela a été aussi l'occasion de réfléchir aux différences et aux complémentarités entre recherche académique et recherche associative et, enfin, de commenter plus directement le périmètre du rapport Duclert.

${ }^{1}$ Doridant Raphaël et Graner François (2020), L'État français et le génocide des Tutsis au Rwanda, Marseille, Agone. 


\section{Le génocide des Tutsi : un évènement hors-normes et ses sources documentaires}

Dans votre récent ouvrage avec Raphaël Doridant, une bonne partie des documents cités émanent de la base de données construite par Jacques Morel. Pouvez-vous revenir sur son élaboration et la façon dont vous l'utilisez ?

Cette base de données est un outil exceptionnel pour documenter le rôle de la France dans le génocide des Tutsi. Au départ, Jacques Morel (qui a longtemps été membre de l'association Survie) avait collecté beaucoup de données et écrit ce très gros livre: La France au cour du génocide des Tutsi ${ }^{2}$. II y avait beaucoup de matière dans ce livre, avec des niveaux de fiabilité inégaux. Sorti en 2010, sa réception a été limitée car il était avant tout destiné à des spécialistes plutôt qu'au grand public. Depuis, la version disponible gratuitement en ligne est régulièrement mise à jour. Lorsque j'ai écrit mon premier livre, mon but était d'extraire $10 \%$ du livre de Jacques Morel, soit la partie la plus convaincante, avec les sources les plus solides, pour qu'elles puissent être connues du grand public ${ }^{3}$.

Jacques Morel étant lui-même informaticien, il a pensé que c'était important qu'une source citée dans son livre puisse être consultée directement via un site web. C'est fantastique: vous cherchez un nom, un mot, vous allez à l'index, vous faites ce que vous voulez et vous trouvez tout en ligne. Ou bien si vous avez la version PDF de son livre, vous cliquez sur la référence et vous aboutissez directement sur le lien du document cherché.

Au bout d'un moment sa base de données a tellement grossi qu'il a souhaité l'améliorer et la structurer, et il en a changé le nom. Désormais nommée France Génocide Tutsi (FGT), elle comporte un outil de recherche qui est très efficace et facile à maîtriser ${ }^{4}$. Ayant accumulé énormément de documents, Jacques Morel se fait à présent aider par Aymeric Givord [membre de l'association de rescapés lbuka]; ils rentrent deux ou trois documents par jour chacun, et c'est beaucoup de travail, car il faut les référencer et les mettre en forme. Ainsi, lorsque vous voulez citer une source dans un ouvrage publié, vous ajoutez le lien vers FGT entre parenthèses, et le lecteur peut facilement y avoir accès s'il veut voir le document.

Ce site a d'ailleurs servi pour les fameuses fuites du fonds Carle, en mettant en ligne à disposition de tout le monde tous les documents.

\section{Justement, pourriez-vous revenir sur le fonds Carle pour celles et ceux qui ne sont pas familiers avec cet épisode?}

Le fonds Carle fait partie des archives de l'Élysée. François Mitterrand, qui travaillait pour I'histoire, a demandé à une collaboratrice, une militante socialiste nommée Françoise Carle, de réunir des documents sur certains sujets : I'Europe ou la Bosnie par exemple. Il s'agissait d'aller voir tous les conseillers du président et de demander à chacun d'eux des sélections

\footnotetext{
${ }^{2}$ Morel Jacques (2010), La France au coeur du génocide des Tutsi, Paris, Izuba/L'Esprit frappeur.

${ }^{3}$ Graner François (2014), Le Sabre et la machette : officiers français et génocide tutsi, Mons, Éditions Tribord.

${ }^{4}$ URL : http://francegenocidetutsi.org/ (consulté le 11 octobre 2021).
} 
d'archives, puis de les photocopier et de les rassembler de manière exploitable. Au cours de ce travail, Françoise Carle a aussi réalisé un dossier sur le Rwanda qui représente deux cartons actuellement aux Archives nationales. Ce sont donc des photocopies de documents existants par ailleurs dans d'autres dépôts, mais une sélection a été opérée par Françoise Carle et les conseillers. Globalement, il y a peu de déchets et beaucoup de choses intéressantes, notamment des comptes rendus des conseils restreints de défense - c'est pourquoi la fuite de ces papiers a permis de mieux comprendre le fonctionnement interne de l'Élysée dans la période 1990-1995.

Voilà le point de départ. II se trouve que ce fonds a ensuite fuité et que les documents ont circulé pendant deux ans parmi des personnes informées, mais sans que ces dernières ne soient vraiment assurées de leur authenticité. Elles ont toutefois commencé à travailler sur le fonds et à l'analyser. Et puis, cela a été versé dans un dossier judiciaire et à partir de ce moment, la justice a été confrontée à un versement d'archives non authentifiées. Les magistrats ont demandé la déclassification de certaines de ces archives qui étaient classifiées, ont reçu les documents authentifiés, déclassifiés, et ont vérifié que c'était les mêmes que ceux ayant fuité. La justice a donc détruit les anciens dès que les nouveaux devenaient disponibles, et elle s'est retrouvée avec un corpus authentifié.

C'est à la suite de cela que ces archives ont commencé à circuler plus largement. En 2010, un article de Rafaëlle Maison en proposait une analyse ${ }^{5}$, puis Jacques Morel les mettait en ligne sur son site et elles devenaient accessibles à tous. On arrivait donc à une situation assez étrange, où officiellement ces archives n'existaient pas et n'étaient donc pas accessibles, mais tout le monde pouvait les lire en ligne. Bruno Boudiguet [ancien membre de Survie] les a réunies, en a enlevé certaines qui étaient inutiles - comme les archives de presse qui effectivement n'étaient pas les plus originales - et en a fait un livre volumineux de près de 700 pages $^{6}$. Là aussi, il n'a eu aucune condamnation, et ce livre permet de citer ces papiers. Ensuite, dès qu'il y a eu plusieurs personnes à même d'accéder à ce fonds Carle aux Archives nationales, la première chose a été de vérifier l'authenticité de l'ensemble, suite à quoi il n'y a eu aucun problème avec la publication de Bruno Boudiguet.

\section{Qu'en est-il des volumes d'archives qui ont émergé de la mission d'information parlementaire (MIP) en 1998, de la commission citoyenne de 2004 ou encore de la commission Mucyo de 2008 ? Comment travailler avec cela et qu'est-ce que cela dit du matériel documentaire disponible?}

Le rapport Mucyo a surtout fait émerger des témoignages qui restent difficiles à recouper. La commission d'enquête citoyenne visait surtout à collecter et recouper des témoignages; elle a contribué à faire évoluer les mentalités et de ce travail est née l'idée de porter plainte. En termes d'archives, c'est surtout la MIP qui a fait avancer les choses.

\footnotetext{
${ }^{5}$ Maison Rafaëlle (2010), « Que disent les "Archives de l'Élysée" ? », Esprit, mai, pp. 135-159.

${ }^{6}$ Boudiguet Bruno (2012), Rwanda. Les archives « secrètes » de Mitterrand, Paris, Aviso/L'Esprit frappeur.
} 


\section{Dans le travail que vous menez à partir de différents types de sources, comment en évaluez-vous la valeur comparée?}

Je pense que, ce qui est important, c'est de se fier à la qualité des sources et non pas à l'identité des auteurs qui les révèlent. La presse a tendance à considérer la fiabilité d'une information uniquement en se basant sur les auteurs de sa production, et non sur leur argumentation. De mon point de vue, c'est un problème. À chaque fois que j'ai une information, écrite ou orale, j'essaie de déterminer sa fiabilité. La personnalité ou l'opinion de l'auteur est l'un des éléments parmi d'autres pour déterminer la fiabilité, ça ne peut pas être le seul. Encore une fois, il y a des gens extrêmement "biaisés » qui, de temps en temps, fournissent des éléments de très bonne qualité. Réciproquement, il y a des gens généralement rigoureux ou fiables, qui ont toute la légitimité ou la crédibilité, mais qui ponctuellement ont un discours soit sans intérêt soit carrément faux. II faut éviter l'argument d'autorité tout autant que l'argument inverse: «Je n'ai pas confiance en cette personne, donc tout ce qu'elle dit est faux. »Bien sûr, cela demande beaucoup de finesse, et c'est pourquoi le recoupement est si important.

Pour mon premier livre, Le Sabre et la machette - qui était plus de la vulgarisation que de la recherche - je partais du principe que ce qui est convaincant, le plus solide, ce que tout le monde va accepter comme argument dans un débat aussi houleux, c'est quand quelqu'un dit quelque chose qui se retourne contre lui. J'avais analysé tout ce que j'ai pu obtenir comme sources publiques (que n'importe quel lecteur pouvait vérifier) émanant de militaires français, et j'avais regardé ce qui confortait les accusations portées contre eux. Pour certaines accusations, je ne trouvais rien, et j'indiquais alors dans mon livre que je ne considérais pas cette accusation comme étayée. Pour d'autres accusations, si tel militaire français s'exprimait contre son intérêt, et si cela confortait exactement l'accusation que portaient des associations ou des rescapés rwandais, on pouvait penser que c'était relativement fiable. Et si je recoupais plusieurs sources différentes qui étaient cohérentes entre elles, alors j'accordais un niveau de confiance assez élevé.

\section{Vous avez beaucoup travaillé avec des témoignages de militaires français, pouvez-vous revenir sur votre méthode ? Par ailleurs, vous êtes-vous appuyé également sur des témoignages de Rwandais, même de manière indirecte ?}

Pour notre livre L'État français et le génocide des Tutsis au Rwanda avec Raphaël Doridant, c'est moi qui ai recueilli l'essentiel des témoignages. Je n'ai pas que des témoignages de militaires. J'en ai interrogé beaucoup mais, l'action de la France n'étant pas uniquement militaire, j'ai interrogé également des personnalités politiques, humanitaires, des personnes de la coopération civile, des fonctionnaires, des journalistes, des gens qui allaient sur le terrain.

J'en ai interrogé certains pendant une minute et d'autres pendant deux heures ou plus... II y a une cinquantaine de personnes citées dans notre livre. En m'appuyant sur la presse ou des livres, j'ai aussi utilisé d'autres témoignages que ceux que j'ai recueillis moimême. Comme je travaille vraiment sur le volet franco-français, je n'ai réalisé que quelques entretiens avec des Rwandais mais j'ai complété avec des témoignages trouvés dans la 
presse. Dans ce cas, le problème du recoupement se pose différemment pour moi, parce que je ne suis pas spécialiste du Rwanda et que je connais moins bien le contexte. Par exemple, quand j'interroge le colonel Tauzin [officier français ayant participé aux opérations Noroît et Turquoise], je connais bien sa classe sociale, son environnement opérationnel, les contraintes auxquelles il était soumis au moment de l'opération, ainsi que les contraintes qui existent au moment où il est en train de me parler. Alors qu'avec d'autres interlocuteurs c'est plus difficile, en particulier s'ils sont Rwandais.

\section{Vous n'êtes jamais allé au Rwanda pour faire un travail de recherche ?}

Non, parce que, pour l'instant, je n'en ai pas eu besoin, d'une part. D'autre part, je rappelle que j'effectue cette recherche en dehors de mon temps de travail principal, essentiellement quand mes enfants sont couchés. C'est pourquoi j'utilise beaucoup d'éléments disponibles sur internet et je réalise des entretiens souvent par téléphone.

\section{Comment articulez-vous l'utilisation d'archives et de témoignages dans votre travail ?}

J'ai recueilli de nombreux témoignages. Il est clair que le témoignage, anonyme ou non, dans un sujet comme celui-là, est vraiment irremplaçable, mais en complément des archives. Les témoignages seuls ne suffisent pas; l'archive seule ne suffit pas. Les deux, ensemble, sont d'une grande richesse. Par exemple, ce qu'on a mis dans notre livre avec Raphaël Doridant couvre la quasi-totalité de ce qu'on a comme sources et, mis à part quelques petits détails, tout ce qu'on a écrit a été recoupé. C'est-à-dire qu'il y a au moins deux sources différentes, en général une écrite et une orale, qui montrent la cohérence et permettent de valider notre propos. C'est extrêmement important pour la validité de notre démonstration. C'est une grande différence entre l'état de la recherche, du savoir, il y a 25 ans, et celui d'aujourd'hui. En effet, l'essentiel de ce que nous écrivons dans le livre était déjà su il y a 25 ans, mais aujourd'hui on a des sources écrites et orales pour le valider.

\section{Archives et recherche associative}

\section{Comment organisez-vous le travail collectif interne à Survie ? Êtes-vous plusieurs à aller voir les archives?}

Avec Raphaël Doridant, qui a une connaissance très profonde et large du sujet, nous avons travaillé en collaboration étroite, jour par jour, et à parts égales. Donc oui, c'est vraiment un travail collectif. Plus généralement notre livre a bénéficié de l'apport ou de la relecture de nombreux bénévoles de Survie. Notre liste de diffusion interne à Survie sur le Rwanda est très active, avec des échanges d'information et des discussions stimulantes. Et plus généralement, de nombreuses personnes d'autres associations ou des journalistes, des chercheurs, des acteurs de l'époque, s'échangent des informations et s'épaulent mutuellement.

Concernant les archives, chaque demande de consultation doit être faite par une personne physique. C'est pourquoi, dans l'affaire de l'ouverture des archives promises par 
Hollande en 2015 (qui n'a pas été complètement suivie d'effet), toute la procédure judiciaire a été entamée suite à une demande que j'avais faite : ça ne pouvait être contesté qu'à titre individuel. Et c'est pourquoi beaucoup de médias se sont focalisés sur mon nom. En réalité le travail juridique a été fait en collaboration avec le cabinet de maître Spinosi et avec l'aide d'Aymeric Givord ; de plus, Survie m'a toujours soutenu. Par ailleurs, les différentes personnes intéressées par la consultation d'archives (que ce soit des Archives nationales ou du Service historique de la Défense à Vincennes - SHD) me contactent souvent. On essaye de se coordonner, bien que tout le monde ne se connaisse pas. Cette semaine [mi-mai 2021] nous commençons justement aux Archives nationales des consultations collectives, par des membres de Survie, d'archives ouvertes suite au travail de la commission Duclert. Donc oui, il y a une concertation.

\section{Quelle distinction faites-vous entre recherche associative et recherche universitaire?}

La recherche associative est souvent militante, tandis que théoriquement la recherche universitaire n'est pas censée l'être. II y a un point commun entre elles, c'est que tout doit être sourcé avec énormément de rigueur. II n'y a aucune concession sur la manière dont on utilise et on cite les informations; la qualité des sources doit être irréprochable, il faut tout vérifier. Mais il y a concrètement aussi de grosses différences, celle du temps disponible en particulier. Quand on est bénévole, on doit faire des choix et cibler ce que l'on cherche, contrairement au caractère exhaustif que permet la recherche académique. Pour le travail dans les archives, c'est très important de pouvoir voir des corpus complets et de faire des analyses exhaustives, mais cette démarche relève plus du domaine académique qu'associatif.

En revanche, le but de l'association Survie étant de faire évoluer la politique de la France en Afrique, notre démarche dans les archives va plutôt consister à mettre en lumière les choses incriminantes. Si l'on trouve ces éléments, il faut de toute façon les mettre en contexte, de la même manière qu'un chercheur universitaire. De ce point de vue d'ailleurs, par rapport à ce qu'a produit la commission Duclert, nous mettons beaucoup mieux en contexte nos informations: par les témoignages, la presse, ou la connaissance du terrain que ne permet pas la seule recherche archivistique. Par moments, le rapport Duclert paraît horssol, alors que notre approche est beaucoup plus documentée et contextualisée.

J'assume le biais consistant à aller dans les archives pour chercher des documents pertinents en peu de temps: je vais balayer très vite pour trouver des informations spécifiques. Par exemple, si je veux savoir si un ordre a été donné de laisser fuir les génocidaires, je vais regarder si ce document existe, en allant directement chercher à la date pertinente, car je connais déjà le contexte. On sait déjà à quelle date le terrain a demandé des instructions, à quelle date l'exécutif en a débattu, et à quelle date l'ordre a été exécuté, il faut donc chercher entre ces deux dates pour voir si l'ordre a été donné par écrit. De même, quand je cherche si le ministre de la Défense a été informé, ou non, de telle opération secrète que les forces spéciales réalisent sur le terrain, je sais à quelle date chercher. Dans d'autres cas, par exemple pour savoir si la France a eu ou non un rôle actif sur les négociations de paix menées par les Rwandais à Arusha, il faut balayer bien plus de documents dans de nombreux 
dossiers et sur une longue période. Au fond, la différence avec la recherche académique, c'est que la question que l'on pose est différente. Cependant, étant universitaire moi-même [François Graner est physicien], j'utilise toutes les méthodes académiques que j'ai acquises professionnellement.

Beaucoup de journalistes nous reconnaissent actuellement comme une source sérieuse, voire la plus sérieuse sur le sujet, grâce à la qualité des informations que l'on produit par exemple dans notre livre. D'autres, parce que l'on est à Survie, refusent de nous écouter ou refusent de nous citer ; c'est une approche qui n'est pas spécifique à ce sujet, d'ailleurs. Dans la presse en général, l'argument d'autorité joue beaucoup, ainsi que la peur de citer une source militante. Cela fait que beaucoup de journalistes reprennent nos informations sans nous mentionner.

Or, pourquoi est-ce que Survie est très critique ? Ce n'est pas par a priori, ce n'est pas une volonté idéologique ou un parti-pris délibéré. C'est parce que la réalité est ainsi : nous faisons des recherches sérieuses, et leurs résultats montrent que les politiques menées sont critiquables. La plupart des membres adhérents de Survie, bénévoles et donateurs, n'ont aucune raison initiale d'être critiques, et moi en particulier. En 1994, j'étais un socialiste bon teint, j'étais un mitterrandien tout à fait respectueux de la République.

\section{Quand vous parlez de « choses incriminantes ", c'est-à-dire que vous espérez que la justice française se saisisse de vos travaux ?}

Ce que j'espérerais avant tout, c'est que la France n'ait rien fait d'incriminant. Une large partie de l'action civile française est peu sous le feu des critiques, même si la politique, la diplomatie, la presse et les coopérants français ont contribué à cautionner une vie politique rwandaise gangrenée par les discriminations et l'extrémisme. En ce qui concerne l'action militaire, les trois opérations françaises au Rwanda ont été complètement duales, avec une face avouable et l'autre inavouable. L'aspect avouable ne pose guère de problème, il y a énormément d'archives là-dessus; nous l'abordons dans notre livre mais nous n'avons pas de raison de nous étendre dessus. Ce que nous voulons faire avancer, c'est la détection des aspects inavouables et leur correction. Cela peut passer par l'action politique, par l'action médiatique, ou par l'action judiciaire. II y a actuellement cinq plaintes pour complicité de génocide contre la France, dont trois soutenues par Survie. Bien sûr, les juges font leurs propres demandes d'archives, mais ce que nous mettons dans notre livre permet d'étayer l'argumentation que nous développons pour contester tout un volet de la politique française. Pour que cela ne se reproduise pas dans le futur.

\section{Quelle distinction faites-vous entre des documents d'archives mis en ligne, et des fonds institutionnels ordonnés et agencés par les services producteurs, à consulter sur papier ? Avez-vous rencontré des problèmes d'authenticité ?}

Évidemment ça n'est pas la même chose. Sur un plan pratique, lorsque vous allez aux Archives nationales consulter par exemple le fonds Delaye [Bruno Delaye, conseiller Afrique de François Mitterrand], vous êtes tributaire du classement, vous devez tout passer en revue ; cela peut être très hétéroclite et pas dans un ordre parfaitement chronologique. Les mêmes 
archives numérisées, vous pourrez y accéder de bien des façons : par date, par sujet, par motclé, etc. Tout ce qu'il y a sur la base de données de Jacques Morel était précédemment sur papier, sauf exception (quelques vidéos ou des articles de journaux), donc l'intérêt de la mise en ligne vient surtout de la facilité de chercher les informations et d'y accéder. Surtout quand un document est numérisé avec reconnaissance de caractères, ce qui permet de faire des recherches automatiques sur des mots contenus dans le document.

Pour ce qui est de l'authentification, elle est souvent plus facile en version papier qu'en version numérique. Cependant, j'ai rarement rencontré de problèmes. Un exemple connu est celui des certificats de décès des deux gendarmes français tués au Rwanda: fausse date, fausse signature de médecin, faux prénom d'un mort, et mention de mort « accidentelle » par «balles d'armes à feu ». Peut-être que simplement quelqu'un paniquait et a fabriqué à la hâte un certificat de décès pour permettre l'évacuation des corps, mais cela contribue à entourer de mystère la mort violente de ces gendarmes. II y a un autre exemple abondamment commenté, c'est celui de l'archive contrefaite qui est citée à la page 295 du rapport Mucyo, concernant le réarmement des génocidaires rwandais qui ont fui au Zaïre ; ce document est truffé d'erreurs et a peut-être été fourni à la commission Mucyo en vue de la discréditer. Cela dit, à ma connaissance il n'y a jamais eu de démenti concernant une archive numérisée sur la base Morel ; ni aucune accusation de « faux ».

II faut évidemment rester vigilant en permanence, mais on a peu de problème d'authentification. En revanche, les distinctions que je fais, pour ma part, sont plutôt de deux ordres : est-ce qu'une archive est fiable ou pas ? Est-ce qu'elle est classée secret-défense ou pas, est-ce qu'elle est publiable légalement ou pas ? En fait, ce qui fait bien plus débat, c'est I'interprétation des archives. Par exemple, quand Hubert Védrine réfute l'article de Rafaëlle Maison, il ne dément pas la source ni le contenu des archives, mais bien l'interprétation qu'elle en fait.

\section{Est-ce que vous pourriez dire un mot sur les dispositions du 7 avril 2021 dans la foulée du rapport Duclert, c'est à dire le décret d'ouverture de certaines archives, ses avancées et ses limites?}

Il faut d'abord dire qu'il s'agit d'une sélection d'archives qui deviennent disponibles, avec les avantages et les inconvénients de la sélection. Ils ont choisi des documents relativement intéressants, même si quasiment aucun n'apporte quelque chose de nouveau. II y a une exception, les éléments montrant qu'au moment du débat pour savoir si la France devait arrêter les génocidaires, les États-Unis lui ont proposé d'adapter le mandat attribué par l'Onu pour que cela devienne possible... et la France a refusé. Voilà, une information vraiment importante, très incriminante pour la France d'ailleurs, mais c'est la seule que je ne connaissais pas et que j'ai trouvée dans le rapport Duclert. C'est d'autant plus important - et cela rejoint mon avis sur le caractère «hors-sol » du rapport - qu'au moment où ces discussions ont lieu, les génocidaires sont déjà partis parce que le Quai d'Orsay les a fait partir. Le rapport ne le mentionne pas du tout, alors que cet élément de contexte est primordial! 
Pour revenir à ce qui devient disponible, c'est donc une sélection. L'avantage est que si vous allez consulter, vous avez essentiellement des documents utiles ou qui ont tel ou tel intérêt. Le gros inconvénient est que ça n'est pas un corpus complet, vous ne savez pas comment la sélection a été faite et quelles sont tous les biais : manque de temps, manque de compétence, manque de volonté ou que sais-je. L'autre gros problème est que ça n'est pas en accès libre. Certes, l'accès est facilité, car il n'y a plus besoin de demander l'autorisation, il faut seulement réserver sa place et y aller. Mais c'est beaucoup plus compliqué que de consulter en ligne. Les archives de la MIP, celles qui ont été publiées, les annexes de la MIP, vous cliquez, vous les avez. Et c'était en 1998. On est en 2021, les nouveaux documents ne sont pas en ligne. Pourquoi ? S'il n'y a rien à cacher, pourquoi ne pas les mettre en ligne ? Pourquoi toujours cette volonté de dissimuler? Officiellement, c'est pour ne pas mettre en ligne d'informations à caractère personnel, mais lesdites informations ont déjà été occultées dans les archives consultables sur papier.

Actuellement [mi-mai 2021, à la suite des restrictions liées aux conditions sanitaires imposées par l'épidémie de Covid-19], aux Archives nationales de Pierrefitte, les places s'arrachent : il faut réserver à partir de minuit chaque nuit et les places disponibles partent en moins d'une demi-heure. Les horaires d'ouverture sont restreints : 23 heures par semaine, pour quelqu'un qui n'est pas francilien, c'est extrêmement difficile d'imaginer aller consulter sur place. On le voit bien, à Survie, c'est ce qui nous limite en ce moment. Pour le SHD, c'est trente heures par semaine, un peu mieux. Et le système de réservation est différent : il faut attendre deux mois pour avoir une place. Donc ça n'est pas très glorieux du point de vue de l'accès aux archives. Pour finir, même celles qui ont été déclarées ouvertes, celles de Balladur par exemple, eh bien quand on va sur le site pour faire une demande, elles sont encore soumises à autorisation. Voilà, entre les erreurs informatiques et les problèmes de communication, cela reste un parcours du combattant, même pour cette toute petite fraction des archives qui a été ouverte. Alors les autres archives au SHD, je ne vous en parle pas?.

\section{Est-ce que c'est selon vous spécifiquement lié au génocide des Tutsi, ou révélateur de la politique archivistique en France?}

Non, pour moi clairement, c'est la culture du secret et des archives en France qui pose problème : c'est une culture du secret-défense. Le secrétariat général de la Défense et de la Sécurité nationale se crispe sur ce sujet-là, il se place au-dessus de la loi et verrouille les archives plus sévèrement qu'il n'a droit de le faire. La question des archives de plus de 50 ans, c'est toute la question de la reclassification du secret-défense ${ }^{8}$. Nous-mêmes, Survie, au titre

\footnotetext{
7 Mi-juillet 2021, Vincent Duclert et Chantal Morelle ont mis en ligne, sur le site du ministère des Affaires étrangères, quelques centaines de documents émanant de ce ministère. URL: https://www.diplomatie.gouv.fr/fr/archives-diplomatiques/actionscientifique-et-culturelle/expositions/expositions-dossiers-en-ligne/collection-de-documents-des-fonds-diplomatiques-francais-

portant-sur-le-rwanda/ (consulté le 11 octobre 2021). Les autres archives consultables mentionnées dans cet entretien, en particulier celles déposées aux Archives nationales ou au Service historique de la Défense, sont maintenant consultables à des horaires d'ouverture plus larges, mais ne sont pas disponibles en ligne. Pour le reste des archives du SHD, c'est-à-dire la majorité, jusqu'à ce jour (11 octobre 2021) toutes les demandes de François Graner se heurtent à des refus.

8 Qui vient de faire l'objet d'une décision du Conseil constitutionnel le 30 juillet 2021. Voir «Après la décision du Conseil constitutionnel du 30 juillet 2021, un débat est nécessaire sur l'accès des citoyens aux archives », Histoire coloniale et postcoloniale, 31 juillet 2021, en ligne. URL: https://histoirecoloniale.net/Apres-la-decision-du-Conseil-constitutionnel-du-30-juillet-2021-undebat-est.html (consulté le 11 octobre 2021).
} 
du génocide des Tutsi, on a contribué à fonder un collectif qui montre comment le secretdéfense pose un gros problème d'enjeu démocratique ${ }^{9}$, sur plein d'affaires où la justice est entravée par le secret-défense ${ }^{10}$. Et même quand les documents ne sont pas secret-défense, le Conseil constitutionnel a montré dans mon affaire ${ }^{11} q^{\prime}{ }^{\prime i l}$ privilégie le secret des gouvernants et non l'intérêt d'informer les citoyens. Heureusement que le Conseil d'État a une position prochel de la Cour européenne des droits de l'homme, qui a une approche plus juridique et plus européenne dans laquelle l'intérêt du citoyen est mis en avant.

Je rappelle d'ailleurs que le secret-défense ne concerne pas exclusivement des sujets militaires, de protection de la défense nationale. C'est la moitié uniquement des documents secret-défense qui sont liés à l'armée. L'autre moitié concerne des sujets purement civils, et il s'agit de la protection des décisions de l'administration et des gouvernants. S'agissant de ce que j'ai vu pour le Rwanda, pour presque tout ce qui a été classifié, on se demande pourquoi ça l'a été : ce sont souvent des documents qui ne contiennent rien de sensible. Parmi les exemples que j'aime citer, il y a un document qui informe : «Monsieur le président, demain je pense aller à la messe en I'honneur du président Habyarimana. Est-ce que vous m'autorisez à y aller? » Ou un autre : "Demain le ministre Léotard ira inspecter nos troupes à l'opération Turquoise. » Bon, c'est secret-défense. Je veux bien que ça le soit pendant 24 heures, mais ensuite, il va y aller avec 200 journalistes, ils sont déjà tous prévenus; en quoi est-ce du secret ? 25 ans après, c'est encore classé secret-défense. C'est incroyable, incroyable.

\section{Combien de temps vous a-t-il fallu pour avoir accès aux archives de l'Élysée, à l'époque ? Quels fonds restent à consulter ?}

François Hollande a promis le 7 avril 2015 que les archives de l'Élysée seraient accessibles à tous les chercheurs avant la fin de l'année suivante. J'ai fait ma première demande peu de temps après. Madame Bertinotti, mandataire de François Mitterrand, m'a d'abord autorisé à consulter une petite partie des dossiers demandés. J'ai fait un recours pour accéder aux autres dossiers : commission d'accès aux documents administratifs (qui a été défavorable), puis tribunal administratif. Et en parallèle j'ai fait une deuxième demande. Pour une raison que j'ignore, la deuxième demande a eu plus de succès, avec près de la moitié des dossiers accordés. Mais le compte n'y était toujours pas et j'ai également fait un recours, commission d'accès aux documents administratifs (qui a été favorable cette fois, sans justifier son changement d'avis), puis tribunal administratif. Le fait que madame Bertinotti n'ait pas à motiver ses avis, qui n'étaient pas susceptibles de recours en justice, a été contesté par une question prioritaire de constitutionnalité (QPC). Le Conseil constitutionnel ayant rejeté cette

\footnotetext{
${ }^{9}$ Collectif «Secret-défense : un enjeu démocratique ». URL : http://collectifsecretdefense.fr/ (consulté le 11 octobre 2021).

10 L'entretien a été réalisé dans le contexte des mobilisations autour de l'instruction interministérielle $n^{\circ} 1300$ (IGI 1300 ) qui imposait une longue procédure de déclassification pour les documents antérieurs à 1970 et portant un tampon « secret ». Si cette instruction contestée a été annulée par le Conseil d'État début juillet 2021, le 30 juillet 2021 le gouvernement a promulgué un nouveau texte de loi rendant accessibles de nouvelles catégories d'archives au bon vouloir des administration productrices - alors qu'elles devraient l'être uniquement sous le contrôle du législateur. Actuellement plusieurs associations d'archivistes et d'historiens s'organisent pour contester ces nouvelles dispositions. Voir Julia Bellot, «Archives: le coup de force de l'été. Entretien avec Noé Wagener", L'Histoire, 10 septembre 2021, en ligne. URL: https://www.lhistoire.fr/archives-le-coup-de-force-de1\%E2\%80\%99\%C3\%A9t\%C3\%A9?fbclid=IwAR0ly_MphoOsVL-KbneTVX-YdjqR_m3IQBBmdNISDFLc3eL3ZC5AEUwG1JI (consulté le 11 octobre 2021)

11 Décision $n^{\circ}$ 2017-655 QPC du 15 septembre 2017, en ligne. URL: https://www.conseilconstitutionnel.fr/decision/2017/2017655QPC.htm (consulté le 11 octobre 2021).
} 
QPC, j'ai saisi la Cour européenne des droits de l'homme. Et j'ai eu le 12 juin 2020 la décision favorable du Conseil d'État, avec des attendus indiquant enfin que l'intérêt du débat public devait primer sur la protection du secret des délibérations gouvernementales... Au total cela a représenté une trentaine d'étapes de procédure, réparties sur cinq ans. J'ai pu consulter les dossiers demandés à l'été 2020.

Ce que j'ai pu consulter n'est qu'une toute petite partie du total. Ce sont les archives de l'Élysée. Ce sont les plus intéressantes puisque l'Élysée décidait. Une autre source importante, ce sont les télégrammes diplomatiques que l'on retrouve aussi bien au ministère des Affaires étrangères qu'à l'Élysée. Mais le principal pour moi maintenant, c'est le SHD de Vincennes. Dans les années passées, les différents demandeurs que je connais n'ont eu que des refus, souvent mal motivés. Cependant, les responsables du SHD que nous avons rencontrés il y a quelques mois nous ont prévenus qu'avec le rapport Duclert, il y aurait des choses qui pourraient changer. Nous refaisons donc une série de demandes et il semble y avoir des frémissements, d'abord parce qu'on a enfin eu accès à l'inventaire. Disons qu'en gros, l'inventaire a été refusé en interne au SHD pour des motifs extrêmement variés et incohérents pendant plusieurs années et ce, jusqu'à la veille de la sortie du rapport Duclert. Son existence même a été parfois niée et finalement, il y a eu une version fuitée par Médiapart. Depuis cette semaine [mi-mai 2021, un mois et demi après la sortie du rapport], on a officiellement I'inventaire du SHD, conforme à ce qui a fuité, à ce qu'on savait.

Aussi, on a reçu les premières réponses positives, partielles mais positives. Dont certaines très curieuses, par exemple l'autorisation de consulter quelques dossiers du fonds François Léotard, que la commission Duclert elle-même n'a pas pu consulter. Cela montre d'une part qu'il y a de l'arbitraire dans les décisions de consultation, et d'autre part, qu'il y a des choses très surprenantes - comme de devoir demander l'avis de François Léotard pour des archives de cette nature, alors que cette décision devrait revenir au ministère des Armées actuel ${ }^{12}$.

Duclert a fait le tour des fonds, ce qui est un énorme travail. Le périmètre qu'il définit est assez correct: le service des archives financières pour Bercy, la Justice, les Affaires étrangères, tout le SHD, la DGSE [Direction générale de la Sécurité extérieure], etc. Le gros problème du rapport Duclert est plutôt la période étudiée, 1990-1994: cette délimitation dans le temps ne permet pas de définir la politique française, entamée bien avant et s'achevant bien après. II y a aussi des archives qui lui ont été refusées (notamment celles de l'Assemblée nationale), et on ne sait pas si d'autres archives ont été écartées ou détruites. Mais dans l'ensemble, les sources archivistiques officielles me semblent assez bien analysées par le rapport.

Après, il y a toutes les archives non officielles, tout ce que les gens ont gardé chez eux, ou tout ce que les unités ont gardé par-devers elles - parce qu'à leur niveau, les unités militaires ne versent pas tout au SHD - et là, il y a une masse de recherches à effectuer pour les historiens. La période commence à être favorable, grâce au tournant provoqué par le

\footnotetext{
12 Depuis la réalisation de l'entretien, François Graner a pu réserver les documents Léotard et une date de consultation en salle. Cependant, deux jours avant la date de consultation prévue, l'autorisation a été annulée. À ce jour (11 octobre 2021), toutes les demandes ont été refusées.
} 
rapport Duclert, qui à la fois ouvre des archives, et rend le climat propice pour que de jeunes historiennes et historiens abordent sérieusement le sujet du rôle de la France au Rwanda.

\section{Le rapport Duclert}

\section{À la lumière des sources que vous savez disponibles, y a-t-il des recherches ou des thématiques que vous ou la commission Duclert n'avez pas pu analyser et que les historiens pourraient explorer plus profondément?}

Pour le rapport Duclert, il faut garder en tête que c'est un travail d'analyse d'archives, de diffusion envers le grand public, et pour ce double rôle il faut saluer leur prouesse, le gros travail mené par la commission en un laps de temps limité. Mais ce n'est pas un rapport de recherche au sens strict. À part quelques détails, il n'apporte rien de nouveau; il est en retrait par rapport à ce qui a été publié par beaucoup de gens, dont Jacques Morel, dont nous, dont d'autres. II ne faut pas considérer qu'il ait mis des choses à jour, à part le refus de la France d'empêcher la fuite des génocidaires.

Alors oui, il reste beaucoup de recherches à faire. Par rapport à tout ce qui a été synthétisé depuis 25 ans, la grosse zone d'ombre, je dirais, c'est le rôle des militaires français et des mercenaires pilotés par l'État français. Leur éventuel rôle, dans l'attentat du 6 avril 1994 [contre l'avion du président Habyarimana] : c'est une piste, lancée dès 1994 par Colette Braeckman et bien d'autres, qui mérite d'être explorée. J'avais fait un article sur la question pour montrer que cette piste ne pouvait pas être exclue a priori, même s'il n'y a aucune preuve de quelque sorte pour l'instant ${ }^{13}$.

Puis, il y a leur rôle pendant le génocide des Tutsi, d'avril à juin 1994, quand il n'y a pas officiellement de soldats français présents en zone gouvernementale. On sait cependant très bien, c'est bien documenté et c'est reconnu par les militaires eux-mêmes, que certains étaient présents, de même que des mercenaires - dont les équipes de Bob Denard et de Paul Barril ${ }^{14}$. On sait qu'il y avait des gens présents, la question de recherche est : qu'est-ce qu'ils ont fait ? C'est encore en discussion.

Et la troisième chose, c'est après le génocide des Tutsi, quand la France aide les exgénocidaires au gouvernement et les forces armées à se réorganiser au Zaïre (actuelle République démocratique du Congo) et qu'ils essayent de se remilitariser pour reconquérir le Rwanda. Là, le rôle des soldats français doit être documenté, parce que c'est encore très parcellaire. II y a deux témoignages qui sont sortis dans Libération hier ${ }^{15}$ mais il y a encore beaucoup de choses à dire là-dessus.

\footnotetext{
13 Graner François (2014), «L'attentat du 6 avril 1994 : I'hypothèse de tireurs et/ou décideurs français vue à travers les textes des officiers français », La Nuit rwandaise, 8, en ligne. URL: https://www.lanuitrwandaise.org/l-attentat-du-6-avril-1994-1,358.html (consulté le 11 octobre 2021).

14 François Crétollier (dir.), «Le crapuleux destin de Robert-Bernard Martin. Bob Denard et le Rwanda », Montreuil, association Survie, février 2018, en ligne. URL : https://survie.org/publications/brochures/article/le-crapuleux-destin-de-robert-bernard-martinbob-denard-et-le-rwanda (consulté le 11 octobre 2021).

15 Maria Malagardis, "Rwanda: Kagame, I'ancien "ennemi", en visite à Paris », Libération, 16 mai 2021, en ligne. URL: https://www.liberation.fr/international/afrique/rwanda-kagame-lancien-ennemi-en-visite-a-paris-

20210514_JPWFVGJVTJCPRPXNV3DGXVICQU/ (consulté le 11 octobre 2021).
} 
Nous nous sommes fait la remarque que le rapport Duclert s'appuyait beaucoup sur les travaux existants sans pour autant les citer. Comment vous positionnezvous par rapport à cela, et comment vous appuyez-vous sur d'autres travaux - il y a quand même toute une littérature sur le génocide des Tutsi ?

De notre côté, nous avons essayé de faire une synthèse de ce qui était su, avec des apports nouveaux: nous essayons de citer toutes les sources qui nous paraissent valables, y compris des gens très militants de tous bords, avec lesquels nous ne sommes pas d'accord du tout. Quand le travail fait est rigoureux, alors on peut tout à fait l'utiliser. II n'y a pas de problème de sélection des sources en fonction des opinions des auteurs, mais bien en fonction de la qualité du matériel qu'ils fournissent.

Cela est effectivement différent dans le rapport Duclert. J'aurais attendu un discours du type de ce qu'on attend en recherche : "Voilà ce qu'on sait déjà, et maintenant nous allons voir si les archives, si deux ans de travail dans les archives, nous permettent d'aller plus loin ». Or leur approche a plutôt consisté à dire: "On va regarder dans les archives ce qu'il y a et puis on va voir si on arrive à la même chose que les autres", ce qui est nettement différent. Mais bon, c'était leur mandat.

Nous qualifions ce rapport à Survie, d'« objet politico-académique ». C'est-à-dire un mélange des deux: ça n'est pas un rapport purement politique, puisqu'il est censé être fait par des gens qui ont une culture académique. Ils ne viennent pas tous de l'enseignement supérieur, mais ils ont une culture de ce type d'analyse. Et puis en même temps, ses objectifs, son mandat sont politiques. On ne leur a pas dit «Faites-nous une recherche universitaire ». Et d'ailleurs, la conclusion (responsabilité accablante de la France, mais pas complicité de génocide) est déconnectée du contenu du rapport: le rapport ne l'étaye pas. Ce qui serait impossible à faire dans un mémoire académique. La conclusion, très vraisemblablement, a été choisie par le pouvoir politique et juxtaposée avec le rapport deux mois avant sa remise officielle au président Macron.

Il y a autre chose de curieux. S'ils avaient été cohérents, ils n'auraient cité aucune autre source que les archives. Dès lors, on aurait presque pu comprendre que la partie du rapport concernant Bisesero ${ }^{16}$ soit resté limitée à ce qu'en écrivent les militaires. Mais comme les membres de la commission Duclert s'autorisent parfois à citer des archives télévisuelles ou des témoignages, alors on se demande, pourquoi ne le font-ils pas tout le temps ? Comment, également, peuvent-ils arriver à des choses aussi absurdes que de parler en long et en large de toutes les archives sur les débats autour de l'arrestation des membres du gouvernement génocidaire, sans évoquer le fait que ces personnes soient déjà parties ? II y a un manque de cohérence dans l'utilisation des sources hors de leur périmètre archivistique.

\footnotetext{
16 Les évènements de Bisesero, où du 27 au 30 juin 1994 des civils tutsi ont été massacrés alors que l'armée française stationnée à quelques kilomètres n'est pas intervenue, sont l'un des principaux points de crispation autour de l'action française au Rwanda pendant l'opération Turquoise.
} 


\section{Pourriez-vous dire deux mots sur la constitution de cette fameuse commission ? Avez-vous été sollicité à un moment ou à un autre?}

Par rapport à la constitution de la commission Duclert, beaucoup a déjà été écrit, notamment sur l'affaire d'Andurain ${ }^{17}$. Pour moi, c'est juste révélateur qu'au moins deux membres de cette commission ont été choisis pour des raisons politiques. Julie d'Andurain avait la légitimité professionnelle pour faire partie de cette commission. Dans les polémiques autour de sa présence, ce qui a été relevé est surtout ce qu'elle a écrit antérieurement sur le génocide des Tutsi, ce qui a pu être interprété comme une apologie du négationnisme. Alors que, pour moi, ce qui posait autant (ou plus) de problèmes, c'est le fait que dans ses écrits antérieurs il y ait des erreurs factuelles et un parti-pris délibéré favorable à l'action de la France. Elle n'était pas neutre, nous l'avons détaillé dans notre article ${ }^{18}$, alors que la commission avait affiché sa volonté d'être neutre. Le second est Christian Vigouroux, qui est un ancien des cabinets mitterrandiens de l'époque. De mon point de vue, sa présence était encore plus problématique que celle de Julie d'Andurain.

Nous avons envoyé notre livre au conseiller Afrique du président Macron, sans aucune réaction, et nous n'avons jamais été sollicités de ce côté. Mais d'autres nous ont sollicités. Par exemple Hervé Berville [député LREM né au Rwanda], avant d'aller au Rwanda pour sa mission [de représentation d'Emmanuel Macron pour la commémoration du génocide en avril 2019], a voulu s'entourer d'un certain nombre de points de vue et il a demandé à Survie de venir le rencontrer. Nous commençons à avoir des sections de certains partis politiques qui nous sollicitent également, Europe Écologie les Verts, des sections du Parti socialiste nous ont demandé de venir faire des interventions explicatives. Bien sûr, il y a d'autres partis, à gauche ou à droite, qui ne veulent pas avoir affaire à Survie.

Notre relation avec la commission Duclert a été un peu surprenante, puisque son président a commencé par dire qu'il allait traiter Survie et France-Turquoise sur le même plan. Or France-Turquoise est l'association créée par des officiers pour se défendre, pour défendre "leur honneur », elle produit un argumentaire en faveur de ses adhérents. Donc, et c'est normal étant donné son objet, elle n'est clairement pas une source d'information, mais bien un groupe de personnes dont les fonctions impliquent un parti-pris. En revanche, les bénévoles de Survie n'ont pas d'intérêt personnel à défendre; Survie produit de l'information, elle est même un des principaux producteurs d'informations, plus avancé que la commission Duclert. Donc, le fait de nous mettre sur le même plan que France-Turquoise était peut-être utile pour Duclert pour se donner une posture de neutralité, ce qui est compréhensible, mais cela a pu le priver de sources pertinentes.

En pratique, Survie a dit à Duclert que nous étions à sa disposition; il est d'ailleurs venu à un colloque que nous avions organisé sur Bisesero et a pu constater notre niveau de connaissance sur le sujet. Finalement, il y a eu une rencontre à sa demande, et cela n'a pas été une réunion de travail, mais un exposé de sa part sur le fonctionnement de la

\footnotetext{
17 Robinet François (2021), « Rwanda 1994 : un rapport pour l'histoire ?», Études, juillet-août, pp. 7-18.

18 François Graner, "Génocide des Tutsis: "I'affaire Julie d'Andurain" », Billets d'Afrique, n 302, novembre 2020, en ligne. URL: https://survie.org/billets-d-afrique/2020/302-novembre-2020/article/genocide-des-tutsis-l-affaire-julie-d-andurain (consulté le 11 octobre 2021).
} 
commission. II n'y a pas eu d'échanges; il ne nous a pas considérés comme fournisseurs de sources. À sa demande, il a également reçu la liste des documents qui ont été refusés à la justice pour cause de secret-défense ; la commission Duclert était, elle, habilitée à consulter les documents secret-défense mais ne semble pas avoir fait usage de cette liste.

\section{Cette séquence terminée, quel vont être les directions et les objectifs de Survie sur ce dossier désormais?}

Du point de vue de Survie actuellement, le rapport Duclert et le rapport Muse ${ }^{19}$ ont l'air calibrés de la même manière, c'est-à-dire qu'ils reviennent le plus possible sur ce que l'on peut reprocher à l'action de la politique française, tout en ne tirant pas les leçons pour changer celle-ci sur le fond. En présentant comme une exception, à la fois l'action de la France au Rwanda, et les mécanismes de pensée et les mécanismes institutionnels qui l'ont permise, ils évitent les questions à se poser pour la suite.

À Survie, nous souhaitons vraiment que ce qui doit être sanctionné soit sanctionné et que les mécanismes soient analysés pour que cela ne se reproduise pas. Aucune leçon n'a été tirée, rien n'empêche que cela se reproduise: la politique africaine de la France, le présidentialisme, cela n'a pas changé sur le fond. D'ailleurs, depuis vingt ans que les élections législatives sont synchronisées sur l'élection présidentielle, et qu'il n'y a plus de cohabitation, le gouvernement et l'Assemblée contrebalancent peut-être encore moins le pouvoir du président qu'à l'époque de Mitterrand.

Pour Survie, si le Rwanda et la France veulent se réconcilier, très bien, mais que cela ne se fasse pas au détriment de la réalité des faits et de la justice, et surtout de la prévention de génocides dans le futur. Pour nous, la prévention est un axe essentiel et nous continuerons les recherches pour essayer de comprendre et faire en sorte que les deux chefs d'État ne puissent pas enterrer le passé comme cela, juste avec un geste symbolique ${ }^{20}$. Je ne suis pas en mesure de dire ce que Survie va décider, mais il me semble vraisemblable que l'action de Survie va continuer d'une part pour obtenir la reconnaissance entière et explicite du rôle de la France au Rwanda, et d'autre part pour modifier les mécanismes qui l'ont rendu possible.

Martin Mourre

IMAF-Condorcet (France)

Florent Piton

IMAF-Condorcet, LabEx HaStec (France)

Nathaniel Powell

Centre for War and Diplomacy, Université Lancaster (Grande-Bretagne)

\footnotetext{
${ }^{19}$ Rapport commandé parallèlement à la commission Duclert par le gouvernement rwandais à un cabinet d'avocats états-uniens : "A Foreseeable Genocide. The Role of the French Government in Connection with the Genocide Against the Tutsi in Rwanda », Washington, Levy Firestone Muse LPP, 19 avril 2021, en ligne. URL : https://www.gov.rw/fileadmin/user_upload/gov_user_upload/2021.04.19_MUSE_REPORT.pdf (consulté le 11 octobre 2021).

20 Quelques jours après cet entretien, la visite officielle d'Emmanuel Macron au Rwanda le 27 mai a donné lieu à un discours du président français et à une conférence de presse conjointe avec son homologue rwandais Paul Kagame.
} 


\section{Bibliographie}

BOUdiguet Bruno (2012), Rwanda. Les archives "secrètes» de Mitterrand, Paris, Aviso/L'Esprit frappeur.

Doridant Raphaël et Graner François (2020), L'État français et le génocide des Tutsis au Rwanda, Marseille, Agone.

GRANER François (2014), "L'attentat du 6 avril 1994 : I'hypothèse de tireurs et/ou décideurs français vue à travers les textes des officiers français", La Nuit rwandaise, 8 , en ligne. URL: https://www.lanuitrwandaise.org/l-attentat-du-6-avril-1994-l,358.html (consulté le 11 octobre 2021).

(2014), Le Sabre et la machette : officiers français et génocide tutsi, Mons, Éditions Tribord.

MAIson Rafaëlle (2010), « Que disent les "Archives de l'Élysée" ? », Esprit, mai, pp. 135-159.

MORel Jacques (2010), La France au coeur du génocide des Tutsi, Paris, Izuba/L'Esprit frappeur.

ROBINET François (2021), « Rwanda 1994 : un rapport pour l'histoire ? », Études, juillet-août, pp. 7-18. 